\title{
Invaginación gastroduodenal por tumor GIST gástrico
}

\author{
Gastroduodenal invagination by gastric GIST tumor
}

Natalia González-Alcolea ${ }^{1}$, María Dolores Chaparro-Cabezas ${ }^{1}$, Félix Martínez-Arrieta ${ }^{1}$ y Víctor Sánchez-Turrión ${ }^{2}$

Mujer de 74 años que acude por melenas y anemia (hemoglobina 9,30 g/dL). Asocia elevación de GGT (255 U/L). La endoscopia alta (Figura 1) objetiva una lesión polipoídea que condiciona invaginación gástrica en el duodeno, que no se consigue reducir. La TC de abdomen (Figura 2) muestra la invaginación, que produce dilatación de la vía biliar, sin dilatación gástrica. Se decide cirugía (Figura
3), realizándose una gastrotomía laparoscópica en la cara anterior del antro, apreciando el pedículo del pólipo, sin conseguir reducirlo. Se convierte a minilaparotomía media supraumbilical ampliando la gastrotomía y reduciendo la lesión, que impresiona depender de la pared, por lo que se realiza una resección en cuña (Figura 4). El estudio anatomopatológico reveló un tumor estromal gastrointestinal
'Hospital Universitario La Paz. ${ }^{2}$ Hospital Universitario Puerta de Hierro Majadahonda Madrid, España.

Recibido el 2 de mayo de 2019 y aceptado para pubicación el 14 de junio de 2019.

Correspondencia a: Natalia González-Alcolea natagoal@hotmail.com
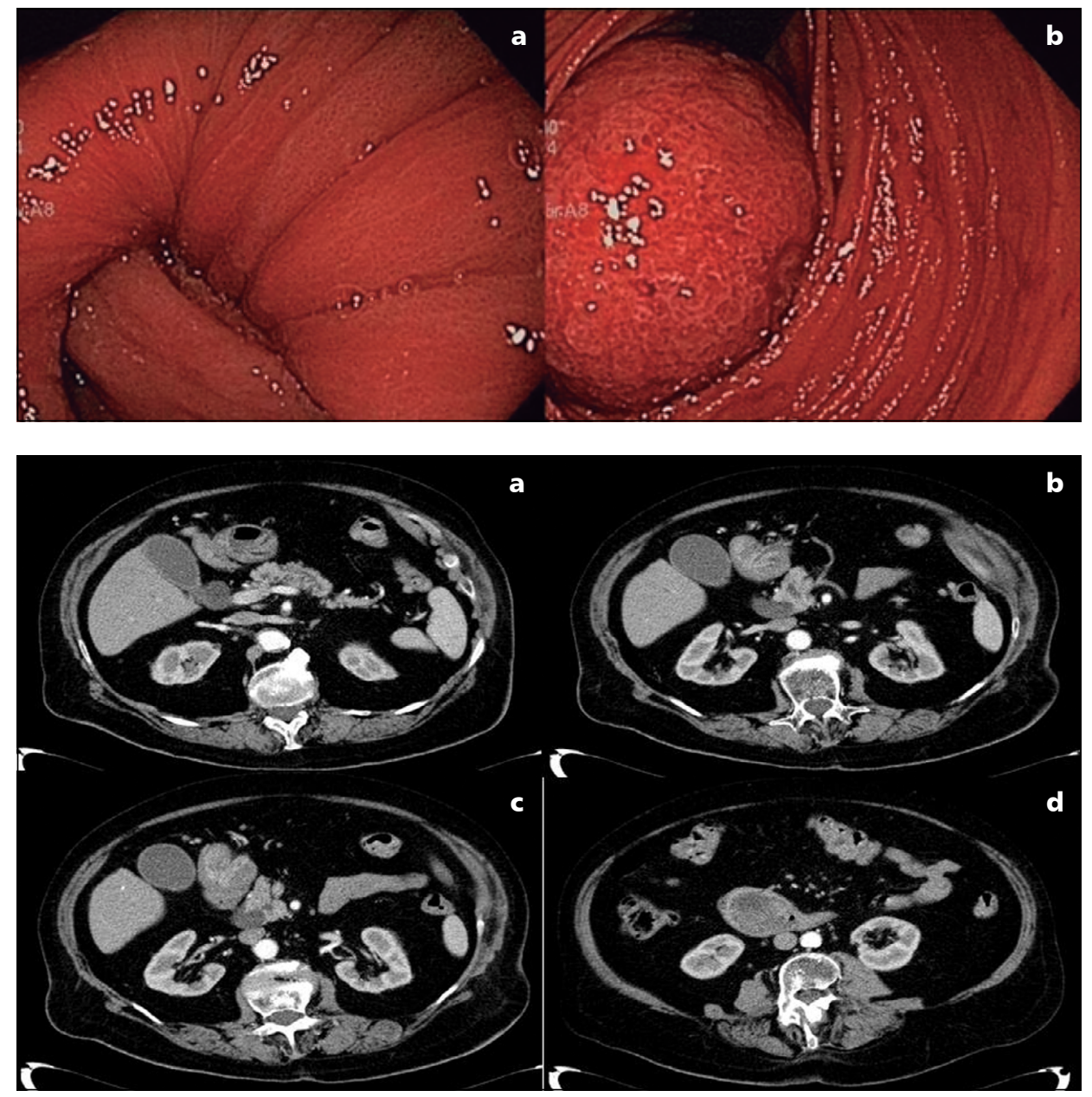

Figura 1. Imagen endoscópica. Se objetiva una zona de tracción desde la cara posterior prepilórica, que se continúa hacia el píloro sugiriendo invaginación duodenal (Figura 1a). Se consigue pasar hasta la segunda porción duodenal, observando en retroversión una lesión polipoidea con algún coágulo, sin signos de sangrado activo ni clara ulceración mucosa, sin ser posible obtener biopsias (Figura 1b).
Figura 2. TC de abdomen con contraste intravenoso. Se aprecia el área de invaginación del estómago en el duodeno (Figuras 2a-b), el pedículo (Figura 2c) y la cabeza del pólipo en el duodeno (Figura 2d). Dilatación de la vía biliar (Figuras $2 \mathrm{a}-\mathrm{c}$ ). 
Figura 3. Cirugía. Gastrotomía en la cara anterior prepilórica (Figura 3a) y tracción del pedículo. La imagen de la derecha corresponde a la lesión una vez ampliada la gastrotomía y reducida por vía abierta (Figura 3b).

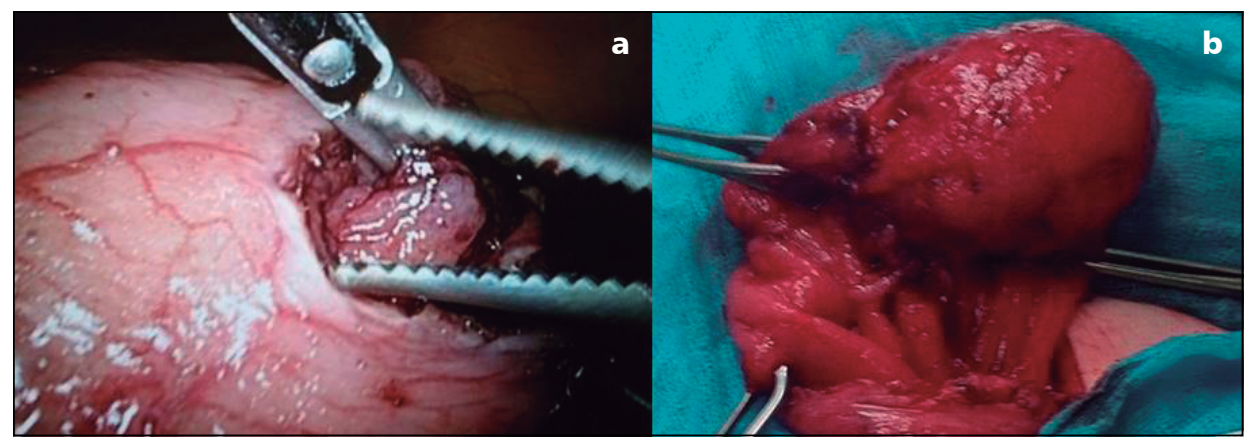

(GIST) de bajo grado histológico de $3 \times 3 \mathrm{~cm}$, sin clara ulceración de la mucosa. El postoperatorio cursó sin incidencias, normalizándose en el seguimiento las cifras de GGT.

Figura 4. Tumor resecado. Reconstrucción de la invaginación gastroduodenal.

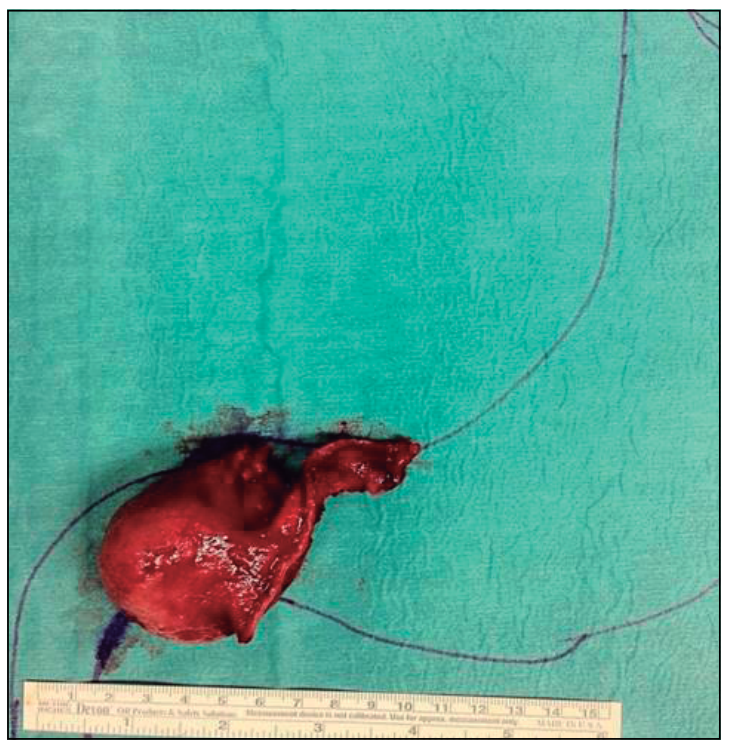

La invaginación gastroduodenal es infrecuente. El $40 \%$ se origina por tumores GIST $^{1}$, de crecimiento lento, causando síntomas por efecto masa; aunque el síntoma más común es el sangrado. Más raro es que ocasionen una invaginación gastrodudenal $^{2}$. En este caso, el diagnóstico se realiza mediante endoscopia y pruebas de imagen (tránsito o TC de abdomen). Suele precisar tratamiento quirúrgico, idealmente laparoscópico ayudado por endoscopia para localizar el pólipo y reducirlo. En los de mayor tamaño puede ser necesaria la laparotomía ${ }^{3}$.

\section{Responsabilidades éticas}

Protección de personas y animales. Los autores declaran que para esta investigación no se han realizado experimentos en seres humanos ni en animales.

Confidencialidad de los datos. Los autores declaran que en este artículo no aparecen datos de pacientes.

Conflictos de interés: no hay.

\section{Referencias}

1. Kadowaki $Y$, Nishimura $\mathrm{T}$, Komoto $\mathrm{S}$, Yuasa T, Tamura R, Okamoto T, et al. Gastroduodenal intussusception caused by a gastric collision tumor consisting of adenocarcinoma and neuroendocrine carcinoma. Case Rep Gastroenterol.
2014;19:89-94.

2. Rittenhouse DW, Lim PW, Shirley LA, Chojnacki KA. Gastroduodenal Intussusception of a Gastrointestinal Stroma Tumor (GIST): Case Report and Review of the Literature. Surg Laparosc Endosc Percutan Tech. 2013;23:e70-e73.
3. Chan CT, Wong SK, Ping Tai Y, Li MK. Endo-laparoscopic Reduction and Resection of Gastroduodenal Intussuception of gastrointestinal Stromal Tumor (GIST): a synchronous endoscopic and laparoscopic treatment. Surg Laparosc Endosc Percutan Tech. 2009;19:e100-3. 